\title{
Ethnologie und Schule
}

Die Ethnologie versteht sich im weitesten Sinne als Wissenschaft, die sich mit der Kultur anderer Länder und Völker auseinandersetzt, wobei man unter Kultur sämtliche vom Menschen geschaffenen Formen versteht, die es ihm ermöglichen, zu überleben. Dazu gehören ebenso der wirtschatliche, soziale und politische Aufbau einer Gesellschaft, als auch Erziehung, Religion, Sitten, Bräuche, Weltanschauungen, Handwerk, Arbeitsteilung usw. Alles, was der Mensch tut, wie und warum er es tut, ist Ausdruck seiner Kultur. Die größtmögliche Vertrautheit verspürt der Mensch in seiner eigenen Kultur, deren Regeln und Gesetze er sich während seines Lebens aneignet. Er kennt die Grenzen und achtet sie, weil er durch willkürliche Grenzverletzungen innerhalb seines Sozialgefüges isoliert wird und vereinsamt.

Andere Kulturen sind ihm fremd, sie verunsichern inn, weil er sich nicht mit ihrer Lebensweise identifizieren kann, weil er verständnislos vor ihren Regeln des Zusammenlebens steht. Die Verflechtungen von Nord-Süd, Zeitungsnotizen, Reiseberichte, die ganze moderne Kommunikation zwingen uns heute allerdings, uns mit anderen Kulturen mehr und mehr zu befassen, denn das Beziehungsnetz zwischen Ländern und Kontinenten verfeinert sich immer mehr, wird komplexer und gespannter,je dichter die Verbindungen wirtschaftlicher, sozialer und politischer Art werden.

\section{Information und ihre Tragweite}

Täglich berichten Radio, Fernsehen und Presse über die Situation in der Dritten Welt. Literatur, Jugendund Kinderbücher erzählen von anderen Völkern mit fremder Lebensart. Aber auch Reiseprospekte, Spielzeugkataloge zeichnen ein bestimmtes Bild fremder Menschen. Oft fällt auf, wie diffus, undifferenziert und kaum hinterfragt solche Informationen und Schilderungen sind.

a) Im Kinderbuch spielen die "Zehn kleinen Negerlein»" ihre große Rolle weiter. «Dr. Doolittle» treibt noch immer sein Unwesen in Afrika und in "Ringgis und Zofis Abenteuern" trägt ein grotesker Eingeborener alle Koffer. Fremde Kulturen werden vielfach verkitscht und verniedlicht, und somit wird beim Kind bereits die Grundlage für das exotisierende und inhumane Bild fremder Kulturen des späteren Erwach- senen vorgeprägt. Die Wirkung solcher Erfahrungen aus der Kinderliteratur ist vermehrt schädlich, weil das Kind kaum genügend kritikfähig ist, um sich gegen die gefährliche Absicht der Erwachsenenwelt zu wehren. Da Kinder- und Jugendbücher heute mehr und mehr als erzieherische Mittel eingesetzt werden, bieten solche Inhalte einen günstigen Nährboden für rassistische Vorurteile, die die Überzeugung des Kindes, da $\beta$ es in der wertvollsten aller möglichen Kulturen lebt, noch verstärken.

$\mathrm{Da} ß$ negative Bilder über fremde Kulturen in Kinderund Jugendbüchern nicht Ausnahme sondern die Regel sind, hat eine Untersuchung dreier Arbeitsgruppen in Bern, Basel und Zürich gezeigt. Alle erhältlichen Jugendbücher über Dritte-Welt-Probleme wurden gelesen und nach bestimmten Kriterien ausgewählt. Etwa $60-70 \%$ der gelesenen Bücher wurden ausgeschieden, da sie den Kriterien nicht standhalten konnten. Die anderen wurden in einem Katalog «Dritte Welt: Empfehlenswerte Kinder- und Jugendbücher» von der "Erklärung von Bern» publiziert. Der Kata$\log$ wird jedes Jahr neu überarbeitet.

b) Spielzeugkataloge: Das Kind spielt als Konsument eine wichtige Rolle. Mehrmals im Jahr verfertigen die Spielzeugindustrien einen farbenprächtigen Katalog, um das Kind über seine Möglichkeiten zu informieren. Dabei spielen vor allem auch Indianer-Figuren aus Plastik eine Rolle, die den üblichen Federschmuck tragen, mit Gewehren auf Weiße knallen und schnelle Plastikrosse reiten. Die Fantasie des Kindes wird in der Spielleitung auch gleich noch kanalisiert, indem man ihm vorzeigt, wie es Kriegssituationen zwischen Rothäuten und Weißen nachspielen kann.

c) Lehrmittel: Geht das Kind zur Schule, wird es mit Lehrmitteln versorgt, die oft versteckt und unterschwellig die gleichen undifferenzierten, ethnozentrischen und intoleranten Aussagen vertiefen. Dazu einige Beispiele:

"Afrika wurde erst spät erschlossen. Noch vor zwei Generationen lebten fast alle Eingeborenen in Stammesverbänden, die von Häuptlingen streng regiert wurden. Ihre Sitten und Gebräuche erschienen dem Europäer primitiv, er fühlte sich weit überlegen. Die Neger trieben Hack-

Dr. Ruth G. Vermot, Schulstelle Dritte Welt, Monbijoustrasse 31, 3011 Bern 
bau, außerhalb des Regenwaldgebietes auch Viehzucht. Alles Land gehörte dem Häuptling oder dem Stamm; jeder erzeugte mit einfachsten Mitteln und Werkzeugen nur so viel, wie er brauchte. Der Trieb zur Arbeit, wie wir ihn kennen, ist beim Afrikaner ursprünglich nicht groß. Erst die Europäer haben wirtschaftliche Arbeitsmethoden und Maschinen eingeführt und Pflanzungen angelegt. Was die Afrikaner bis dahin produzierten Hirse, Reis, Maniok, Yams und einige Ölfrüchte - reichte nur für ihren eigenen Bedarf; für den Weltmarkt hatten ihre Ernten keinerlei Bedeutung. - Heute werden landwirtschaftliche Erzeugnisse in großen Mengen ausgeführt, teils von Plantagen, teils aus kleineren Betrieben der Afrikaner: Bananen, Kakao,..."

"In einem Großteil Afrikas sind die Bewohner Animisten: Für sie ist die Natur mit Geistern besiedelt, die in das Leben der Menschen eingreifen. Der Zauberer des Dorfes ist gleichsam ein Wahrsager, Ratgeber und Arzt.»

"Sie (die Araber) haben den Afrikanern den Islam aufgezwungen und sie jahrhundertelang durch ihre grausamen Sklavenjagden heimgesucht. ... Erst die Europäer haben in den letzten Jahrzehnten einen durchgreifenden Umschwung eingeleitet. Sie haben den Sklavenhandel, der ganze Landstriche entvölkert hatte, lahmgelegt und für Ruhe und Ordnung gesorgt."

In einem neuerschienenen Lehrmittel «Weltgeschichte im Bild", Band 6, zweite überarbeitete Auflage 1977, wird in einem Abschnitt (S. 89) über das Wohnwesen der Völker das abgewandelte Sprichwort verwendet: "Sage uns wie $D u$ wohnst, und ich sage Dir, wer $D u$ bist...». Der Satz ist auf die Bilder aus Afrika, Alaska,

Indonesien und Europa angewendet.

Die Beispiele könnten endlos aneinandergereiht werden. Das Kind nimmt sie bereitwillig auf, und langsam entstehen dann unfertige und erschreckende Bilder bei den Kindern und Jugendlichen, über die Jörg Becker vor einiger Zeit in seiner Studie «Zur Darstellung peripherer Gesellschaften im Kinder- und Jugendbuch", einer Untersuchung über die Stereotypen beim jüngeren Schüler über verschiedene Länder und Kulturen, berichtete:

\section{AFRIKA}

Dunkler Kontinent, riesige Urwälder, Tiere, Tierparks, primitive Eingeborene, seltsame Riten und Tänze der Eingeborenen, Jagd, Safari, nackte Neger, moderne Großstädte, akademisch gebildete Afrikaner.

\section{ARABIEN}

Islam, Schleier tragende Frauen, Harem, Blutrache, schnelle Pferde, heimlicher Weingenuß, stolze und freiheitsliebende Nomaden, geheimnisvolle Stadt Mekka, Wüstensöhne.

\section{INDIEN}

Maharadschas in Seidengewändern, Brillanten, Göttin Kali, Fakire, religiöser Fanatismus, Pilger, Benares, Kasten, Armut, Tempel, märchenhafte Pracht, Tiger, Jagd.

\section{LATEINAMERIKA}

Inka, Maya, Cortez, Pizarro, Kolumbus, Giftpfeilindianer, Amazonas, Urwald, Lamas, Banditen, moderne Großstädte, Armut, Deutsch im Urwald.

\section{INDIANER}

Prärie, Pferde, Büffel, Federschmuck, Zelte, Häuptlinge, Medizinmann, Cowboys, Ausrottung durch die Weißen.

$\mathrm{Da}$ diese Stereotypen nicht Ausnahme sind, zeigt auch die Schülerumfrage in Ulm, Deutschland:

Fragen aus einer Schülerumfrage in Ulm 1973:

Was ist Ihre Meinung zu folgenden Sätzen?

- Die Leute in den Entwicklungsländern sind nicht fähig zur Selbstregierung.

- Die Menschen in den Entwicklungsländern sind zu faul.

- Die Menschen in den Entwicklungsländern sind weniger intelligent.

- In den Entwicklungsländern herrscht Hunger und Armut vor allem, weil die Menschen dort primitive Vorstellungen haben.

Rund jeder dritte Schüler in Gymnasien und Realschulen (35\%) und jeder zweite Schüler an Berufsschulen (51\%) hält die Menschen in den Entwicklungsländern entweder nicht zur Selbstregierung fähig oder bestätigt ihnen Faulheit, geringere Intelligenz oder primitive Vorstellungen.

(Lüpke/Pfäfflin: Abhängige Dritte Welt, Laetare-Imba, Seite 27.)

Solche stereotypen Bilder beim Kind und beim Erwachsenen sind unumgänglich, wenn man bedenkt, daß Bildungsinhalte immer kulturspezifisch sind und sich stets an den geltenden Normen und Regeln der eigenen Gesellschaft orientieren, die ihrerseits die Kontinuität der Gesellschaft gewährleistet und optimale Schutzmechanismen für die eigene Lebensweise darstellen. Andere Kulturen halten dem Vergleich mit der eigenen nicht stand und sind konsequenterweise stark ethnozentrisch und somit minderwertig dargestellt.

\section{Schulrealität und Dritte-Welt-Problematik}

Die Aufgabe des Ethnologen besteht darin, solche Mechanismen und ethnozentrische Inhalte mit adäquaten Mitteln bewußt zu machen. Dabei müssen vor allem die Lehrer und Schüler sein Zielpublikum sein. Hier muß er eine Möglichkeit suchen, immer wieder aufzuzeigen, wie andere Kulturen funktionieren, welche Sozialgefüge existieren, welche Aufgaben den Menschen zukommen, wie andere Menschen ihre Kon- 
flikte lösen, wie ihre Arbeitsteilung oder die wirtschaftliche Zusammenarbeit aussieht usw. Er muß aber gleichzeitig aufzeigen, welche Mechanismen die Entwicklungsländer von den Industrieländern abhängig halten, warum es einen Nord-Süd-Dialog gibt, wie Befreiungsbewegungen arbeiten, was Selbsthilfe-Organisationen sind, wie der Weltmarkt funktioniert und weshalb die Reichen reicher, die Armen ärmer werden. Diese Informationsarbeit muß nun in den Schulalltag transferiert werden, und hier beginnen die großen Schwierigkeiten, nämlich mit der Frage: Wie kann ich denn bei Lehrern und Schülern Interesse für alle diese Probleme wecken? Lehrer, die sich für diese Fragen engagieren, geben zu, daß sich die Dritte-WeltProblematik für die Schule «eigne», da sie dem Schüler die Relativität der eigenen Werte und Normen vor Augen führen kann.

Indem man sie mit fremden Kulturen konfrontiert, zeigt man den Schülern die Lebensweise anderer Kulturen. Das kann dazu führen, daß die Schüler die eigenen Werte nicht mehr bedingungslos akzeptieren, sondern kritisch abwägen. Die vertiefte Kenntnis über andere Kulturen führt den Schüler wiederum dazu, seine eigene Kultur aus einem anderen Blickwinkel zu betrachten und eine kritische Distanz zu seiner eigenen Lebensweise zu gewinnen. Mit kritischer Distanz ist nicht Ablehnung der eigenen Kultur verknüpft, sondern besseres Erfassen der Zusammenhänge, Toleranz der eigenen und anderen Kulturen gegenüber. Somit kann auch eine Basis für solidarisches Verhalten anderen Völkern gegenüber geschaffen werden.

Sehr viel Mutlosigkeit tönt aber aus den Urteilen der meisten Lehrer, die sich beklagen, daß heute der Schüler kaum mehr für solche komplexen Probleme wie das der Dritten Welt zu motivieren sei, er würde sich viel mehr angesprochen fühlen von den Problemen aus seinem konkreten Lebensbereich. «Töffli», Berufswahl, Sportgeschehen, Schulangst, Leistungsdruck usw. stehen dem Schüler sehr viel näher als die Lebensweise anderer Völker, und ihre Probleme, die entstehen aus Ausbeutung und Abhängigkeit durch die Industrieländer.

Für den Ethnologen lautet die Frage: Welche Bezugsmöglichkeiten zum Problembereich Dritte Welt bietet der alltägliche Erfahrungsraum der Schüler und auch der Lehrer? Wo liegt die Möglichkeit, betroffen zu machen, und wo kann man Raum schaffen, um dieses Betroffensein leben zu können?

Während man auf der Vorschul- und Unterstufe noch viel leichter die emotional-affektive Ebene einbeziehen und mit einfachen Fakten und Bildern arbeiten kann, um dem Kind alltägliche Zusammenhänge aufzuzeigen, um sie mit eigenen Erfahrungen zu verbinden, muß man sich in der Mittel- und Oberstufe sehr viel eingehender mit der viel komplexeren Erfahrungswelt des bereits emanzipierten und selbständigeren Schülers auseinandersetzen, um Anknüpfungspunkte zu finden. Die konkreten Lebensumstände müssen dabei im Vor- dergrund stehen, selbst auf die Gefahr hin, daß die großen entwicklungspolitischen Zusammenhänge nicht vollständig aufgezeigt werden können. Die Verhältnisse in der Dritten Welt und die Beziehungen der Entwicklungsländer zu den Industrieländern standen bisher viel zu sehr im Mittelpunkt. Dabei wurde übersehen, daß «ein Lernen in den Konfliktfeldern der Ersten Welt Voraussetzung einer Sensitivierung für die Dritte Welt ist» (Peter Schläpfer 1977, Dritte Welt im Unterricht. Zürich).

Problematisieren von Themen der Dritten Welt heißt, von politischen und sozialen Zwängen ausgehen, Ungerechtigkeiten in der eigenen Umgebung aufdecken, Fragen aufwerfen, die den Schüler in seinem täglichen Leben belasten, denn «nur da, wo es gelingt, in kleinen, unmittelbar konflikt-orientierten Befreiungsversuchen wenigstens einmal das Bewußtsein möglicher Autonomie herzustellen, könnte es zu einer aktiven Sensitivierung auch für Konflikte und Leiden anderer kommen. Ohnmacht (...) läßt sich nicht durch das Bewußtsein von Ohnmacht aufheben, sondern nur durch die (...) entgegengesetzte Erfahrung wiedererrungener eigener Macht (...), realer Fähigkeit (...), also gelungener eigener Lebenspraxis» (Gronemeyer/ Behr 1977, Erwachsenenbildung: Testfall Dritte Welt. Opladen).

Wichtig ist hier also das Aufzeigen von Handlungsmöglichkeiten in der eigenen Umwelt. Die Möglichkeiten der Schule sind hier allerdings eher gering, da die Schule vom konkreten Lebensprozeß stark getrennt ist und auch ein Lehrer bald einmal in den Ruf der einseitigen politischen Beeinflussung der Schüler gerät, wenn seine Klasse öffentliche Aktionen durchführt, die über das Sammeln von Spendengeldern hinausgehen.

Unterricht, der sich von der Erlebniswelt des Schülers ableitet, verlangt auch eine Planung und Strukturierung des Unterrichts, bei der die Schüler miteinbezogen werden und Schwerpunkte mitbestimmen können.

Die Realisierung eines solchen Unterfangens hat sich seit jeher als schwierig herausgestellt, da die Schule von einigen Ausnahmen abgesehen - tiefgreifende Experimente ablehnt, die Veränderungen provozieren könnten. Unterrichtsmaterialien, die bis heute erschienen sind, halten sich meist an die Forderungen der traditionellen Schule und enthalten kaum Vorschläge über einen alternativen Unterricht. Einige Versuche (Hinweise auf Möglichkeiten wie Rollenspiele Interviews, Theater und Simulationsspiele, alternative Texte usw.) genügen nicht. Ethnologen, die sich mit der Erarbeitung von Schulmaterial befassen, sollten sich nicht ausschließlich um Fakten kümmern, sondern vor allem auch versuchen, Elemente von $z$. B. jeux dramatiques, Pantomime, themenzentriertes Schulspiel (Heinrich Werthmüller, Zürich, «Kaktus») so zu benutzen, daß sich Lehrer und Schüler davon angesprochen fühlen.

Motivieren und Einbeziehen des persönlichen Lebensbereichs reichen allerdings nicht aus, denn Lehrer und 
Schüler müssen sich vor allem auch an die Gegebenheiten der Institution Schule mit ihren Regeln und Gesetzen halten.

\section{Gesamtlehrplan als Möglichkeit, die Dritte- Welt-Problematik in der Schule eingehend zu erfahren}

Problematisch sind nicht nur Motivation der Schüler und richtige didaktische Verarbeitung, sondern auch die vorhandenen Strukturen, nämlich die Schule als Institution mit den Imperativen der Lehrpläne, dem fixen Fächerkanon usw. Wichtig für den Ethnologen und seine Arbeit mit Lehrern und Schülern ist daher auch die Einsicht in die Lehrpläne aller Stufen. Welche Fächer werden unterrichtet? Welche Inhalte werden vermittelt? Wie lassen sich neue Inhalte in den bestehenden Stoff eingliedern, ohne die Stoffülle noch $\mathrm{zu}$ vergrößern? Noa Zanolli, Ethnologin aus Bern, ist vor einiger Zeit diesen Fragen in einem Arbeitspapier nachgegangen. Sie versucht, die Schulrealität einzubeziehen, die u. a. aus einem fixen Fächerkanon mit variierbaren Inhalten besteht. Ihr wichtigstes Postulat ist, daß Dritte-Welt-Probleme nicht mehr in Randstunden, Intensiv- oder Landschulwochen verdrängt werden, sondern daß alle Fächer aller Stufen geeignet sind, daß der Lehrer den Komplex Dritte Welt «kontinuierlich, systematisch und auf eine umfassende Weise angeht». Es geht dabei um eine «Gesamtschau, um ein Gesamterleben der mannigfachen und sich überschneidenden Bezugssysteme (Wirtschaft, Politik, Wissenschaft, Technik, Kunst, Weltanschauung usw.) und damit verbunden um die Vermittlung der Erkenntnis, daß der Komplex «Wir und die Dritte Welt» bzw. auch Zweite Welt eben nicht zwei bzw. drei nebeneinander her sich bewegende Welten bilden, sondern daß unser soziales Milieu die gesamte Welt geworden ist, bzw. miteinschließt».

Das Ziel besteht darin, «uns von unserer anspruchserhebenden Weltsicht zu lösen, eine Konzeption aufzubauen, die von dieser neuen sozialen Realität ausgeht, die einerseits das internationale Beziehungsnetz in den Vordergrund stellt und andererseits die Pluralität der Kulturen achtet, ohne daß dadurch Solidarität gegenstandslos würde». Weiter sollen auch Methoden entwickelt werden, die eine Verwirklichung der Zielsetzung - «Gesamtschau, Ermöglichung der Vertrautheit des uns zunächst fremden und Veränderung unsererVerhaltensweisen» - zulassen. Diese Verwirklichung kann erreicht werden, «indem man so früh als möglich, so lang andauernd als möglich und so umfassend als möglich die Vermittlung von Wissen und Erfahrung in diesem Fragenkreis angeht bzw. in den Schulunterricht einbaut».

Es geht nicht darum, «außerhalb eines bestimmten kantonalen Lehrplans zu arbeiten, sondern innerhalb der bestehenden Lehrpläne zusätzliche Erkenntnisse zu vermittlen»".

Weiter geht es nicht darum, irgendwelche Stundenzahlen vorzuschlagen, die gewissermaßen absolviert werden sollen, sondern lediglich für jedes Fach und für jede Stufe Möglichkeiten aufzuzeigen, wie der gesamte Komplex miteinbezogen werden könnte, welche Hinweise zu geben sind, welches zusätzliche Material verwertet werden könnte.

Zum methodischen Vorgehen sagt sie:

«Es ginge nun darum, einen Katalog von Konzepten bzw. Erkenntniszielen aufzustellen, die soweit möglich der Forderung der Gesamtschau entgegenkommen wie zum Beispiel:

- Menschen leben auf der ganzen Welt

- Alle Menschen haben Grundbedürfnisse

- Die natürliche Umwelt des Menschen ist begrenzt

- Menschen verwerten ihre Umwelt auf verschiedene Weise

- Der Mensch hat überall bedeutende schöpferische Fähigkeiten (Initiativen, Erfindungen) in sämtlichen Bereichen (soziale Organisationsformen, Sprache, materielle Kultur) entwickelt.

- Die Ursache von Armut gründet nicht auf Faulheit

- Eine hoch entwickelte Wirtschaft bedeutet nicht glückliche Menschen

- usw.»

Aufgrund dieser Erkenntnisziele müßten nun in jedem Fach und für jede Stufe Themen gesucht werden, die sich in den Unterricht einbauen ließen, z. B.

- Menschen leben auf der ganzen Welt und haben trozt gleicher Grundbedürfnisse verschiedene Lebensweisen entwickelt. (Vgl. Lehrmittel «Wie Menschen leben».)

Dieses Erkenntnisziel kann zum Beispiel wie folgt in den Unterricht einbezogen werden:

Fach Sprachen Lesen von Texten, die die Lebensweise anderer Völker miteinbeziehen

Unterstufe, Mittelstufe Verse, Erzählungen, Rätsel, Sprichworte usw.

Fach Mathematik Stoff zur Darstellung mathematischer Sachverhalte aus anderen Kulturen, z. B. Makalla-Spiel

Literatur: «Africa counts, Numbers and Pattern in African Culture», Zaslavsky, 1973

Heimatunterricht Wohnhaus bei uns - Wohnhaus in anderen Regionen (Grundbedürfnis Wohnen) Garten bei uns - Gärten in anderen Ländern (Grundbedürfnis Nahrung). Quartier Dorf (Grundbedürfnis Gemeinschaft)

Lebenskunde Wie sind wir organisiert: Ghettoisierung der Alten, Kranken, Geistesgestörten, Marginalen, Waisenkinder usw. Wie lösen andere Kulturen diese Probleme? 
Geographie

Oberstufe
Umweltprobleme bei uns. Wie verhalten sich die Menschen anderer Kulturen ihrer Umwelt gegenüber? Was bedeutet sie ihnen/mir? (Siehe z. B. Rede des Indianerhäuptlings Seattle).
Die meisten Fragen über Ethnologie und Schule sind zwar ungelöst, aber in stetem Gespräch.

In der Schweiz wie auch in anderen deutschsprachigen Ländern werden verschiedene Anstrengungen unternommen, dem Lehrer Unterrichts- und Arbeitshilfen anzubieten.

\section{Ethnologie und Schule:}

\section{a) Museen}

Das Völkerkundemuseum in Zürich bietet den Schulen ein eigenes Schulzimmer an, Schaudepots und einen Mehrzwecksaal. Gemeinsam mit dem Pestalozzianum werden Kurse für Lehrer, die in Ausbildung stehen, durchgeführt. Arbeitsgruppen (Lehrer) erarbeiteten Unterrichtseinheiten über Prärie- und Plains-Indianer und über die Indianer der Nordwestküste. Nach einem Kurs über Tibet sollen nun ebenfalls Unterrichtseinheiten entstehen, die Ende 1979 veröffentlicht werden. Die Unterrichtseinheiten entstehen im Baukastensystem, so daß der Lehrer zu verschiedenen Zeitpunkten verschiedene Aspekte der Kulturen aufzeigen kann. Gleichzeitig sollen Tondokumente, Diaserien und eine eigene Sammlung von Duplikats- oder Repliksobjekten entstehen, die an Schulen ausgeliefert werden.

Neben diesen Aktivitäten spielen Lehrerberatung, Führungen und Wechselausstellungen im Museum eine wichtige Rolle in der Zusammenarbeit zwischen Ethnologie und Schule.

Das Völkerkundemuseum in Basel ist im Umbau, plant aber für 1980 eine Ausstellung über die Abellam (NeuGuinea), die die Sozialisation eines Abellam-Kindes zum Thema hat. Die Ausstellung ist speziell für die Schule gedacht, sollen doch auch gleichzeitig Unterrichtseinheiten über dieses Thema publiziert werden. Kinder- und Jugendführungen werden trotz Umbau weitergeführt (Bau eines indianischen Drachen, Kleidung aus Tüchern usw.).

\section{b) Schulstelle Dritte Welt, Bern}

Seit drei Jahren führt die Schulstelle Dritte Welt in Bern vermehrt Lehrerfortbildungskurse durch, die dem Lehrer ermöglichen, nicht nur andere Kulturen kennenzulernen, sondern in denen die Entwicklungspolitik einen wesentlichen Raum einnimmt. Immer werden zu solchen Kursen Ethnologen beigezogen, denn Kulturen und deren Zerstörung durch Kulturzusammenprall sind eine wesentliche Diskussions- und Erfahrungsbasis innerhalb dieser Kurse.

Die Schulstelle Dritte Welt bietet Lehrern Unterrichtsmaterialien, Materialpakete zu verschiedenen Themen und Kinder- und Jugendbücher an, die ihnen helfen sollen, ein anderes und gerechteres Bild anderer Kulturen zu zeichnen.

Während des Internationalen Jahres des Kindes (IJK) 1979 sollen in der ganzen Schweiz verschiedene Aktionen (Unterrichtseinheiten zu Kinderbüchern, Tagungen, Ausstellungen usw.) durchgeführt werden, Adressaten sind wiederum die Lehrer.

Ein weiteres Anliegen der Schulstelle Dritte Welt ist die Erarbeitung alternativer Methoden und Didaktik, die viel mehr als bisher die Einheit des ganzen Menschen befürworten und die Kopflastigkeit des aktuellen Unterrichtes vermindern.

\section{c) Workshop der Universität Zürich}

Noa Zanolli führte im Sommersemester 1978 im Rahmen der Ethnologie einen Workshop durch mit dem Titel «Aufbereitung ethnologischen Materials für Schule und Öffentlichkeit». Das Hauptanliegen dieses Workshops war, daß Studenten entweder einzeln oder in Gruppen ethnologisches Material so aufbereiten, daß es allgemeinverständlich wird und sich für die Arbeit in Schule und Öffentlichkeit eignet. Bedingung für die Mitarbeit war unter dieser Voraussetzung der Wille, wirklich zu produzieren, zu publizieren - damit sollte eine «Trockenübung» umgangen werden.

Für die Schule wurden drei verschiedene Unterlagen erarbeitet:

aa) Der Schulfunk übertrug eine Sendung mit dem Titel «Indianer - einst und heute».

bb) Eine Gruppe startete eine Untersuchung in Schaffhausen innerhalb der Kindergärten, Primar-, Sekundar-, Mittelschulen und Lehrerseminarien über die Problematik der ethnologischen Themen im Unterricht. Die Resultate dieser Bestandesaufnahmen sollen in der Schaffhauserzeitung veröffentlicht werden.

cc) Tonbildschau über die australischen Ureinwohner, erarbeitet von einer Studentin, die längere Zeit in Australien verbracht hat. 